\title{
What is the added value of combined core biopsy and fine needle aspiration in the diagnostic process of renal tumours?
}

\author{
K. Barwari • I. P. Kummerlin • F. J. ten Kate • \\ F. Algaba $\cdot$ I. Trias $\cdot$ H. Wijkstra $\cdot$ J. J. De la Rosette $\cdot$ \\ P. Laguna
}

Received: 4 February 2011/Accepted: 12 May 2011/Published online: 28 May 2011

(c) The Author(s) 2011. This article is published with open access at Springerlink.com

\begin{abstract}
Purpose Non-diagnostic results still hinder the routine use of core biopsy (CB) and fine needle aspiration (FNA) in the diagnostic process of renal tumours. Furthermore, substantial interobserver variability has been reported. We assessed the added value of combining the results of $\mathrm{CB}$ and FNA by five pathologists in the ex vivo diagnosis of renal mass.

Methods Two ex vivo core biopsies were taken followed by two FNA passes from extirpated tumours. All samples were evaluated by five blinded pathologists. A consensus diagnosis of the surgical specimen was the index for comparison. For each pathologist, the number of nondiagnostic (non-conclusive or undetermined biology and failed biopsies), correct and incorrect scored cases of each technique was assessed. When a non-diagnostic CB or FNA had a correct diagnostic counterpart, this was considered as of added value.
\end{abstract}

K. Barwari ( $)$ · I. P. Kummerlin · H. Wijkstra .

J. J. De la Rosette · P. Laguna

Department of Urology, AMC University Hospital,

University of Amsterdam, Amsterdam, The Netherlands

e-mail: k.barwari@amc.uva.nl

\section{F. J. ten Kate}

Department of Pathology, AMC University Hospital,

Amsterdam, The Netherlands

F. Algaba

Pathology Department, Fundació Puigvert,

Universitat Autònoma de Barcelona, Barcelona, Spain

I. Trias

Pathology Department, Hospital Plató, Barcelona, Spain
Results Of the 57 assessed tumours, 53 were malignant. $\mathrm{CB}$ was non-diagnostic in 4-10 cases (7-17.5\%). FNA established the correct diagnosis in 1-7 of these cases.

FNA was non-diagnostic in 2-6 cases (3.5-10.5\%), and the counterpart CB established the correct diagnosis in 1-6 of these cases.

For the 5 pathologists, accuracy of $\mathrm{CB}$ and FNA varied between $82.5-93 \%$ and $89.5-96.5 \%$, respectively. Combination of both types of biopsy resulted in 55-57 correct results (accuracy 96.5-100\%), i.e., an increase in accuracy of $3.5-14 \%$.

Conclusion Combining the result of $\mathrm{CB}$ and FNA in renal mass biopsy leads to a higher diagnostic accuracy. Recommendations on which technique used should be adapted to local expertise and logistic possibilities.

Keywords Renal cell carcinoma - Aspiration biopsy Needle biopsy · Kidney neoplasm · Pathology

\section{Introduction}

Most renal tumours are incidental findings discovered by cross-sectional or ultrasound imaging, and these imaging techniques are also the current pre-operative 'gold standard' to determine the characteristics of the renal tumour. However, most studies have also shown that differentiating benign from malignant renal mass based on radiological features is difficult, and subtype differentiation of renal cell carcinoma (RCC) is highly uncertain [1].

In contrast to suspected malignancies in other organs, pre-operative biopsy to confirm the pathology has not been widely adopted in renal tumours. Fear of potential tract seeding and low accuracy of the test were the most important caveats. However, tract seeding has not been 
reported since 1994, and the accuracy of renal tumour biopsy is reaching $90 \%$ and higher in some set-ups [1, 2]. Main indications for biopsy in renal masses are clinical T1 masses, suspicion of metastasis from other primary cancer or infectious disease and before systemic targeted therapy.

The majority of the published studies deal with the results of core biopsies ( $\mathrm{CB}$ or Tru-cut biopsies). Fine needle aspiration (FNA) is less frequently reported, although some studies show similar accuracy compared with CB [1]. Survey studies show that if indicated, the majority of practicing physicians prefers core biopsy (CB) over FNA. Both techniques have their specific advantages and disadvantages. Histological core biopsy is thought to preserve the architecture of the tumour and facilitates the performance of ancillary tests and immunohistochemistry, possibly benefitting accuracy. On the other hand, it is more likely to harvest cells that lead to a diagnosis when multiple passes are made through the tumour, as is done when taking an FNA [3-6].

Very few studies have assessed the diagnostic accuracy of a combination of CB and FNA (e.g. Wood et al. [7]) or the superiority of one technique over the other. Therefore, the clinical question remains, when performing a renal mass biopsy, which technique is the first choice [8]. The aim of the present study is to asses the accuracy of combining both techniques and the added value of either of the techniques.

\section{Materials and methods}

Fifty-seven consecutively extirpated renal masses were included in this prospective study. Since no direct intervention was made in these patients, informed consent was not mandatory.

Immediately after surgery, the fresh specimen was studied at the pathology laboratory. First one urologist performed under direct vision two CBs from the periphery of the tumour with an automatic 18-gauge core biopsy system (Boston Scientific () ). The CBs were fixed in formalin, paraffin embedded and haematoxylin-and-eosin (HE)-stained. Subsequently, the same urologist performed fine needle aspirations of the tumour using a 22-gauge needle. After each of two passes, two smears were stained for Giemsa and two for Papanicolaou. After FNAs and CBs were performed, the surgical specimen was processed according to the guidelines of the Uropathology Working Group (European Society of Pathology) [9] and the European Working Group of Uropathology of the European Association of Urology.

All samples were evaluated by five pathologists blinded for the definitive diagnosis and in an independent manner. The pathologists classified the biopsies and smears according to the latest WHO criteria. Samples containing only normal kidney parenchyma, blood, necrotic tissue or insufficient tumour tissue to make a definitive diagnosis (according to the individual pathologist's assessment) were classified as non-diagnostic. Samples in which the pathologist could not decide between malignant or benign were classified as non-conclusive. Because no diagnosis could be made in these samples, for the purposes of this article, they will also be considered non-diagnostic.

After the blind evaluation of the FNAs and CBs was completed, the five pathologists reached consensus on the surgical specimen at the multi-head microscope. The specimen diagnosis was considered the standard reference to assess the diagnostic accuracy of each of the techniques and the combination of both. Three of the pathologists worked in an academic reference centre and two in community hospitals. All pathologists had experience with uropathology, renal CBs and general cytology.

The standard reference and the results of the $\mathrm{CB}$ and FNA were classified as malignant, benign or a non-diagnostic result. Results were considered accurate when it was diagnostic and the diagnosis corresponded with the result of the standard reference in terms of a benign or malignant mass. Subsequently, we considered the two biopsy techniques together as (one single) index test. A case with one or both outcomes malignant was considered as a positive index test, consequently a case with both outcomes being benign and/or non-diagnostic was considered as a negative index test (see Table 1).

We analysed the number of correct outcomes of the index test, i.e., a positive index test with a positive reference standard or a negative index test with a negative reference standard outcome.

\section{Results}

Patient data are presented in Table 2. Of the 57 renal masses, four tumours (7.0\%) were benign and $53(93.0 \%)$ were malignant.

For the different pathologists, CB was incorrect or nondiagnostic in 6-11 cases $(10,5-19,3 \%)$ with FNA leading to the correct diagnosis in $0-10$ of these cases $(0-17,5 \%)$.

Table 1 Construction of the index test $(\mathrm{CB}+$ FNA)

\begin{tabular}{lll}
\hline Test 1 & Test 2 & Index test \\
\hline $\mathrm{M}$ & $\mathrm{M}$ & $\mathrm{M}$ \\
$\mathrm{M}$ & $\mathrm{B}$ & $\mathrm{M}$ \\
$\mathrm{M}$ & $\mathrm{ND}$ & $\mathrm{M}$ \\
$\mathrm{ND}$ & $\mathrm{ND}$ & $\mathrm{ND}$ \\
$\mathrm{B}$ & $\mathrm{ND}$ & $\mathrm{B}$ \\
$\mathrm{B}$ & $\mathrm{B}$ & $\mathrm{B}$ \\
\hline
\end{tabular}

$M$ Malignancy, $B$ Benign tumour, $N D$ Non-diagnostic result 
Table 2 Patient data

\begin{tabular}{ll}
\hline Number of tumours & 57 \\
Mean age (years) (SD) & $61.9(13.2)$ \\
Mean tumour size in cm (SD) & $5.4(2.6)$ \\
Gender (\%) & \\
Male & $36(63.2)$ \\
Female & $21(36.8)$ \\
Side (\%) & \\
Left & $22(38.6)$ \\
Right & $32(56.1)$ \\
Not known & $3(5.3)$ \\
Operation (\%) & \\
Radical nephrectomy & $39(68.4)$ \\
Partial nephrectomy & $18(31.6)$ \\
Pathological diagnosis of surgical specimen $(\%)$ & \\
Clear cell RCC & $43(75.4)$ \\
Chromophobe RCC & $4(7.0)$ \\
Papillary RCC & $3(5.3)$ \\
Renal oncocytoma & $2(3.5)$ \\
Angiomyolipoma & $2(3.5)$ \\
RCC unclassified & $1(1.8)$ \\
Urothelial carcinoma & $2(3.5)$ \\
\hline
\end{tabular}

$S D$ Standard deviation, $R C C$ Renal cell carcinoma

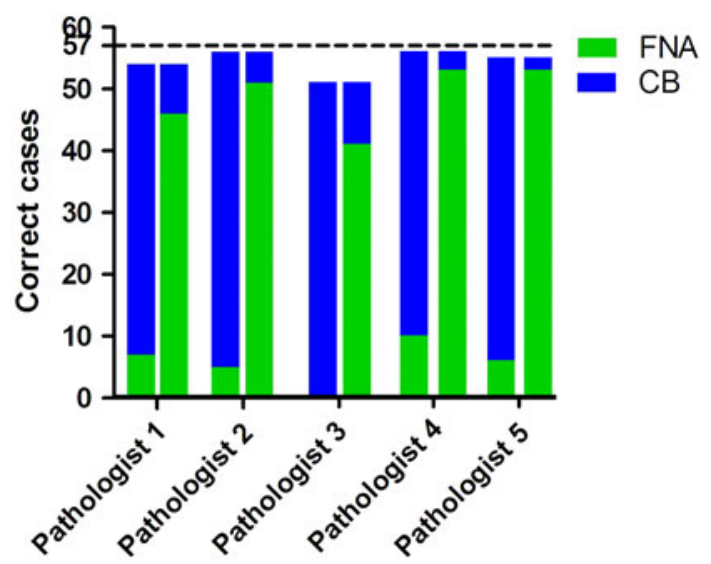

Fig. 1 Added value of CB and FNA for every pathologist. Total no. of tumours is represented by the dashed line $(n=57)$. The first bar for every pathologist shows the added value of FNA (in green). The second bar for every pathologist shows the added value of $\mathrm{CB}$ (in blue)

FNA was incorrect or non-diagnostic in 4-16 cases (7,0-28,1\%) with $\mathrm{CB}$ leading to the correct diagnosis in $2-10$ of these cases $(3,5-17,5 \%)$ (see Fig. 1).

Results per pathologist

Pathologist 1 had $10(17,5 \%)$ non-diagnostic CBs and 6 $(10,5 \%)$ non-diagnostic FNAs.
Table 3 Accuracy for CB, FNA and CB + FNA combined

\begin{tabular}{llll}
\hline Pathologist & $\begin{array}{l}\text { Correct CB } \\
(\%)\end{array}$ & $\begin{array}{l}\text { Correct FNA } \\
(\%)\end{array}$ & $\begin{array}{l}\text { Correct } \\
\text { CB+FNA (\%) }\end{array}$ \\
\hline 1 & $47 / 57(82.5)$ & $46 / 57(80.7)$ & $54 / 57(94.7)$ \\
2 & $51 / 57(89.5)$ & $51 / 57(89.5)$ & $56 / 57(98.2)$ \\
3 & $51 / 57(89.5)$ & $41 / 57(71.9)$ & $51 / 57(89.5)$ \\
4 & $46 / 57(80.7)$ & $53 / 57(93.0)$ & $56 / 57(98.2)$ \\
5 & $49 / 57(86.0)$ & $53 / 57(93.0)$ & $55 / 57(96.5)$ \\
\hline
\end{tabular}

CB was concordant with the reference standard in 47 cases $(82,5 \%)$, FNA in 46 cases $(80,7 \%)$. When combining $\mathrm{CB}$ and FNA, there was concordance in 54 cases leading to an accuracy of the combined biopsy of $94,7 \%$.

Pathologist 2 had $6(10,5 \%)$ non-diagnostic CBs and 3 $(5,3 \%)$ non-diagnostic FNAs.

CB was concordant with the reference standard in 51 cases $(89,5 \%)$, FNA in 51 cases $(89,5 \%)$. When combining $\mathrm{CB}$ and FNA, there was concordance in 56 cases leading to an accuracy of the combined biopsy of $98,2 \%$.

Pathologist 3 had 4 (7\%) non-diagnostic CBs and 3 $(5,3 \%)$ non-diagnostic FNAs. There were 2 cases with a non-diagnostic result for both $\mathrm{CB}$ and FNA (reference standard: clear cell RCC and chromophobe RCC, respectively). $\mathrm{CB}$ was concordant with the reference standard in 51 cases $(89,5 \%)$, FNA in 41 cases $(71,9 \%)$. When combining $\mathrm{CB}$ and FNA, there was concordance in 51 cases leading to an accuracy of the combined biopsy of $89,5 \%$.

Pathologist 4 had $8(14,1 \%)$ non-diagnostic CBs and 2 $(3,5 \%)$ non-diagnostic FNAs.

CB was concordant with the reference standard in 46 cases $(80,7 \%)$, FNA in 53 cases $(93,0 \%)$. When combining $\mathrm{CB}$ and FNA, there was concordance in 56 cases leading to an accuracy of the combined biopsy of $98,2 \%$.

Pathologist 5 had $8(14,1 \%)$ non-diagnostic CBs and 3 $(5,3 \%)$ non-diagnostic FNAs. There was 1 case with a nondiagnostic result for both CB and FNA (reference standard: chromophobe RCC).

CB was concordant with the reference standard in 49 cases $(86,0 \%)$, FNA in 53 cases $(93,0 \%)$. When combining $\mathrm{CB}$ and FNA, there was concordance in 55 cases leading to an accuracy of the combined biopsy of $96,5 \%$.

In summary, the accuracy of $\mathrm{CB}$ to detect a malignant tumour ranged from 80.7 to $89.5 \%$ for the different pathologists and the accuracy of FNA ranged from 71.9 to $93.0 \%$. When both techniques were combined, the accuracy ranged from 89.5 to $98.2 \%$ (Table 3).

\section{Discussion}

Renal mass biopsy as a diagnostic tool has gained renewed interest among urologists due to changes in a number of 
involved fields. First, the incidence of incidentally discovered SRM increased significantly in the era of widely accessible imaging modalities and since it is recognised that these SRMs represent a heterogeneous group of both benign and malignant pathological conditions with only a minority bearing high-grade tumours [10] Simultaneously, advances in surgical management have been made facilitating less aggressive treatment modalities for less aggressive tumours, such as thermal ablation of even active surveillance [11]. Nowadays pathologists have access to techniques such as immunohistochemistry and molecular diagnostics to determine biopsy tissue with higher accuracy [12].

In previous studies, the accuracy of $\mathrm{CB}[13,14]$ and FNA has been assessed individually; however, no recommendation to perform the one or the other was given [8]. Since both techniques have different advantages and disadvantages, we think one technique can be complementary to the other.

In this study, we assessed the added value of $\mathrm{CB}$ on FNA and vice versa in determining the biology of a renal mass by five different pathologists. The consulted pathologists were practicing at the time of the study in different hospitals varying from academic centres to community hospitals, and therefore, we covered a wide clinical spectrum and integrated the inter-observer variability which is demonstrated to be substantial by Kümmerlin et al. $[13,14]$.

In our setting, the overall accuracy of $\mathrm{CB}$ and FNA is comparable with figures reported in the recent literature [1, 2] with the accuracy of CB ranging from 80,7 to $89,5 \%$ between the different pathologists and the accuracy of FNA from 71,9 to $93,0 \%$. However, in this study, no immunohistochemistry has been used, which most likely would have improved the figures.

The second aim of this study was to demonstrate the superiority of CB over FNA or vice versa. However, we found comparable accuracy figures for both $\mathrm{CB}$ and FNA (80,7-89,5\% for CB and 71,9-93,0\% for FNA). Since accuracy figures of both techniques are approaching each other, it is hard to proclaim to be either superior or complementary.

What type of biopsy to perform depends on the local (logistical) situation. When striving for the highest accuracy and minimising the risk of non-diagnostic biopsy results, it is recommended to use both biopsy techniques. However, when the given setting has a known high accuracy of one of the two techniques, performing this one only one is recommended in order to save time and resources.

We recognise some limitations of this study. First, the biopsies were taken in an ex vivo setting. Clinical application of biopsies will be an in vivo (percutaneous) setting presumably leading to lower accuracy figures. However, a comprehensive recent review comparing accuracy figures of ex vivo- and clinical biopsy studies showed comparable if not better results of percutaneous biopsies in a clinical setting [2], and therefore, a direct extrapolation of the ex vivo results might be expected. Second, the mean tumour size in the current study is larger than in SRM in which biopsy might have major application. However, the mean tumour size in our series represents the contemporary mean size at surgery including T1b renal masses, $10 \%$ of which may still be benign [10] justifying biopsy in this range of tumour size as well. Furthermore, case selection was not based on clinical premises as the present study was designed to assess the additional value of either of the biopsy techniques over a single biopsy. Third, due to the ex vivo nature of the study, an additional risk of complications related to the extra biopsies performed cannot be ruled out. However, large series in the modern literature of $\mathrm{CB}$ and FNA reported very few or no major complications, and minor complications such as post-biopsy bleeding are very uncommon and almost never of clinical significance $[6,7$, $15,16]$.

Lastly, additional biopsy will lead to increased costs in the clinical setting. In our ex vivo setting, the additional FNA biopsy would have represented an incremental cost of $247 \%$ (core biopsy analysis $€ 85.49$ compared with $€ 299.47$ for analysing an FNA, including $€ 187.17$ for on-site analysis by a pathology analyst). Ultimately, a proper costbenefit analysis including in vivo procedural costs, costs of complications and cost-benefit in terms of preventing unnecessary surgical interventions should be carried out before definitive inclusion of additional biopsies in the diagnostic algorithm.

\section{Conclusion}

Accuracy of $\mathrm{CB}$ and FNA is high in this ex vivo setting, and adding the results of $\mathrm{CB}$ to FNA and vice versa resulted in an accuracy ranging from 89,5 to $98,2 \%$ for the different pathologists. Whether to perform both $\mathrm{CB}$ and FNA depends on the performance of CB and FNA solely and on the logistical possibilities in the particular setting. Both techniques should be performed when striving for minimal non-diagnostic and maximal accurate results.

Acknowledgments We would like to thank colleagues Dr. F. Smedts and Dr. T. Horn for their collaboration in pathological assessment.

Conflict of interest No competing financial interest exists.

Open Access This article is distributed under the terms of the Creative Commons Attribution Noncommercial License which permits any noncommercial use, distribution, and reproduction in any medium, provided the original author(s) and source are credited. 


\section{References}

1. Volpe A, Jewett MA (2009) Current role, techniques and outcomes of percutaneous biopsy of renal tumors. Expert Rev Anticancer Ther 9:773-783

2. Lane BR, Samplaski MK, Herts BR, Zhou M, Novick AC, Campbell SC (2008) Renal mass biopsy-a renaissance? J Urol 179:20-27

3. Kümmerlin IPED, ten Kate FJW (2008) Biopsies and cytological punctures. In: Renal cell carcinoma: diagnosis and therapy. Springer, New York

4. Khan AA, Shergill IS, Quereshi S, Arya M, Vandal MT, Gujral SS (2007) Percutaneous needle biopsy for indeterminate renal masses: a national survey of UK consultant urologists. BMC Urol 7:10

5. Kummerlin IP, Borrego J, Wink MH, Van Dijk MM, Wijkstra H, de la Rosette JJ, Laguna MP (2007) Nephron-sparing surgery and percutaneous biopsies in renal-cell carcinoma: a global impression among endourologists. J Endourol 21:709-713

6. Schmidbauer J, Remzi M, Memarsadeghi M, Haitel A, Klingler HC, Katzenbeisser D, Wiener H et al (2008) Diagnostic accuracy of computed tomography-guided percutaneous biopsy of renal masses. Eur Urol 53:1003-1011

7. Wood BJ, Khan MA, McGovern F, Harisinghani M, Hahn PF, Mueller PR (1999) Imaging guided biopsy of renal masses: indications, accuracy and impact on clinical management. J Urol 161:1470-1474

8. Ficarra V, Novara G (2009) Editorial comment on: cytological punctures in diagnosis of renal tumours: a study on accuracy and reproducibility. Eur Urol 55:196
9. Lopez-Beltran A, Bassi PF, Pavone-Macaluso M, Montironi R (2004) Handling and pathology reporting of specimens with carcinoma of the urinary bladder, ureter, and renal pelvis. A joint proposal of the European Society of Uropathology and the Uropathology Working Group. Virchows Arch 445(2):103-110

10. Frank I, Blute ML, Cheville JC, Lohse CM, Weaver AL, Zincke $\mathrm{H}$ (2003) Solid renal tumors: an analysis of pathological features related to tumor size. J Urol 170:2217-2220

11. Kunkle DA, Uzzo RG (2008) Cryoablation or radiofrequency ablation of the small renal mass: a meta-analysis. Cancer 113:2671-2680

12. Yang XJ, Sugimura J, Schafernak KT, Tretiakova MS, Han M, Vogelzang NJ, Furge K et al (2006) Classification of renal neoplasms based on molecular signatures. J Urol 175:2302-2306

13. Kummerlin I, ten Kate F, Smedts F, Horn T, Algaba F, Trias I, de la Rosette J et al (2008) Core biopsies of renal tumors: a study on diagnostic accuracy, interobserver, and intraobserver variability. Eur Urol 53:1219-1225

14. Kummerlin IP, Smedts F, ten Kate FJ, Horn T, Algaba F, Trias I, Wijkstra $\mathrm{H}$ et al (2009) Cytological punctures in the diagnosis of renal tumours: a study on accuracy and reproducibility. Eur Urol 55:187-195

15. Neuzillet Y, Lechevallier E, Andre M, Daniel L, Coulange C (2004) Accuracy and clinical role of fine needle percutaneous biopsy with computerized tomography guidance of small (less than $4.0 \mathrm{~cm}$ ) renal masses. J Urol 171:1802-1805

16. Wunderlich H, Hindermann W, Al Mustafa AM, Reichelt O, Junker K, Schubert J (2005) The accuracy of 250 fine needle biopsies of renal tumors. J Urol 174:44-46 